\title{
Freiwilliges Engagement - Bedeutung für Gesellschaft und Politik
}

\author{
Corinna Kausmann, Nadiya Kelle, Julia Simonson \& \\ Clemens Tesch-Römer
}

Im Jahr 2019 sind 39,7 Prozent der Bevölkerung ab 14 Jahren in Deutschland freiwillig engagiert - rund 28,8 Millionen Menschen. Es sind Menschen unterschiedlichen Alters und Geschlechts, Menschen aus unterschiedlichen beruflichen Kontexten, Menschen mit und ohne Migrationshintergrund, Menschen aus verschiedenen Bundesländern. Die Vielfalt der freiwillig engagierten Menschen spiegelt die Vielfalt der Gesellschaft in Deutschland wider. Freiwillig Engagierte trainieren Kinder und Jugendliche in Sportclubs, sie organisieren Veranstaltungen in der Gemeinde, sie leisten Nachbarschaftshilfe für ältere Menschen, sie übernehmen ehrenamtliche Unterstützung in Krankenhäusern, sie sind in Schülervertretungen engagiert oder sie setzen sich in Bürgerinitiativen für ihre Kommune ein.

\subsection{Unterschiede und Ungleichheiten im freiwilligen Engagement}

Freiwilliges Engagement ist eine Form der gesellschaftlichen Partizipation und ein bedeutsamer Faktor für die soziale Integration in die Gesellschaft (Alscher et al. 2021; Roth 2020). Freiwilliges Engagement geht mit sozialen Kontakten einher sowie mit der Möglichkeit, eigene Erfahrungen und Kenntnisse einzubringen und neue Kenntnisse und Fertigkeiten zu erwerben (Düx et al. 2009). Interpretiert man freiwilliges Engagement in diesem Sinne als wichtige Teilhabemöglichkeit, so ist es erstrebenswert, allen Menschen in der Gesellschaft die Chance zu ermöglichen, ein freiwilliges Engagement aufzunehmen. Der Deutsche Freiwilligensurvey 2019 zeigt aber, dass es erhebliche Unterschiede in der Ausübung und Ausgestaltung freiwilligen Engagements gibt. Geschlecht, Alter, Bildung und Migrationshintergrund sind mit zum Teil deutlichen Unterschieden und Ungleichheiten im freiwilligen Engagement verknüpft. 
Geschlecht. Zwischen Frauen und Männer lassen sich - trotz ähnlicher Engagementbeteiligung im Jahr 2019 - deutliche Unterschiede in der Ausgestaltung des freiwilligen Engagements ausmachen. Frauen engagieren sich beispielsweise anteilig häufiger als Männer in Bereichen, die als familienbezogen oder sozial charakterisiert werden können. Männer sind hingegen eher in der Politik, bei Unfall- oder Rettungsdiensten sowie in der freiwilligen Feuerwehr engagiert. Zudem werden Leitungsfunktionen im Engagement anteilig häufiger von Männern als von Frauen ausgeübt, und freiwillig engagierte Männer wenden anteilig häufiger mehr Zeit für ihre freiwillige Tätigkeit auf als Frauen. Diese Geschlechterunterschiede können in den noch bestehenden Mustern der geschlechtsspezifischen Arbeitsteilung begründet sein, wobei Frauen häufiger als Männer die Doppelaufgaben von Familie und Beruf zu bewältigen haben. Darauf deuten auch die Gründe für die Beendigung eines freiwilligen Engagements hin: Bei Frauen fallen familiäre Gründe deutlich stärker ins Gewicht als bei Männern. Gleichstellungspolitische Maßnahmen könnten helfen, Frauen und Männern nicht nur den Zugang, sondern auch die Ausgestaltung des freiwilligen Engagements gleichermaßen zu ermöglichen und Barrieren abzubauen.

Alter. Die höchsten Anteile Engagierter weisen Personen im jüngeren und mittleren Erwachsenenalter auf. Personen zwischen 50 und 64 Jahren engagieren sich zu etwas geringeren Anteilen und der geringste Anteil Engagierter ist bei den Personen ab 65 Jahren zu finden. Diese unterschiedliche Beteiligung kann unter anderem auch in den Anknüpfungspunkten für freiwilliges Engagement begründet sein, die von der jeweiligen Lebensphase und von den Lebensumständen abhängig sein können. Auch die Ausgestaltung des Engagements kann sich je nach Lebensphase unterscheiden: Es zeigt sich, dass vor allem ältere Engagierte eine zeitintensive freiwillige Tätigkeit ausüben oder auch eine Leitungsposition übernehmen. Es ist davon auszugehen, dass Engagierte im jüngeren und mittleren Erwachsenenalter aufgrund ihrer zeitlichen Inanspruchnahme durch Beruf und Familienverpflichtungen anteilig seltener Leitungsfunktionen sowie zeitintensives Engagement ausüben. Politische Maßnahmen, die die Vereinbarkeit von Beruf, Familie und Engagement in den Blick nehmen, können die Menschen bei der Beteiligung im Engagement und bei der Ausgestaltung des Engagements unterstützen.

Bildung. Menschen mit höherer Bildung sind anteilig häufiger engagiert als Menschen mit niedriger Bildung. Dieses Ergebnis kann darauf hindeuten, dass die Zugangschancen zum freiwilligen Engagement für Menschen mit hoher Bildung besser sind als für Menschen mit niedriger Bildung. Die ungleichen Zugangschancen können darin begründet sein, dass Menschen mit höherer Bildung sich leichter Informationen zur Aufnahme und Ausübung eines freiwilligen Engagements verschaffen können. Zudem verfügen sie in der Regel über ein höheres Einkommen als Personen mit niedriger Bildung, was die Aufnahme und die Ausübung eines freiwilligen Engagements begünstigt. Durch ein höheres Einkommen besteht oft ein größerer 
finanzieller Spielraum für Ausgaben, wie beispielsweise Mitgliedsbeiträge in Vereinen oder Fahrtkosten, die Voraussetzungen für freiwillige Tätigkeiten sein können. Allerdings ist auch festzustellen, dass sich Personen mit niedriger Bildung besonders zeitintensiv engagieren und dass sich keine Unterschiede hinsichtlich der Ausübung von Leitungs- und Vorstandsfunktionen zwischen den Bildungsgruppen zeigen. Für die Engagementpolitik könnte dies bedeuten, vor allem die Zugangschancen zum freiwilligen Engagement für Menschen mit niedriger Bildung zu verbessern. Bildungsunterschiede scheinen vor allem bei der Aufnahme einer freiwilligen Tätigkeit eine Herausforderung zu sein, da die Unterschiede in der Ausgestaltung geringer sind. So haben möglicherweise Kenntnisse und Fähigkeiten, die im Rahmen der freiwilligen Tätigkeit erworben wurden, bei der Ausübung der Leitungsfunktionen mehr Gewicht als die schulische Bildung einer Person.

Migrationshintergrund. Zwischen Menschen mit Migrationshintergrund und Menschen ohne Migrationshintergrund zeigen sich erhebliche Unterschiede in der Engagementbeteiligung. Menschen mit Migrationshintergrund sind deutlich seltener freiwillig engagiert als Menschen ohne Migrationshintergrund. Einen großen Unterschied gibt es auch bei Leitungs- und Vorstandsfunktionen: Personen mit Migrationshintergrund üben in ihrer freiwilligen Tätigkeit anteilig seltener eine leitende Position aus als Personen ohne Migrationshintergrund. Besonders gering ist der Anteil Engagierter mit einer leitenden Tätigkeit bei Personen mit eigener Zuwanderungserfahrung, die keine deutsche Staatsangehörigkeit haben. Sprachbarrieren sowie ein unterschiedliches Verständnis von freiwilligem Engagement könnten Gründe für die unterschiedliche Ausübung von Leitungspositionen sein (Vogel et al. 2017). Aber auch Vorbehalte durch zivilgesellschaftliche Institutionen sowie Diskriminierung und Rassismus können die Ausgestaltung freiwilliger Tätigkeiten einschränken oder auch einen negativen Einfluss auf die generelle Beteiligung am Engagement haben (Beigang et al. 2016; Schirilla \& Schramkowski 2012). Integrationspolitische Maßnahmen können Menschen mit Migrationshintergrund den Zugang zum freiwilligen Engagement erleichtern und auch Gelegenheiten ermöglichen, Leitungs- oder Vorstandsfunktionen im Engagement zu übernehmen.

Im Zeitvergleich sind einige der Unterschiede und Ungleichheiten im freiwilligen Engagement in den letzten zwanzig Jahren zurückgegangen. So hat sich die Engagementbeteiligung von Frauen und Männern über die Zeit hinweg angeglichen: Im Jahr 2019 zeigen sich erstmals seit 1999 keine statistisch signifikanten geschlechtsspezifischen Unterschiede. Die Anteile freiwillig Engagierter sind in allen vier betrachteten Altersgruppen seit 1999 gestiegen, allerdings in einem unterschiedlichen Ausmaß und einer unterschiedlichen Dynamik. Der größte Zuwachs zeigt sich dabei für die 65-Jährigen und Älteren, auch wenn sie weiterhin im Vergleich der Gruppen die geringste Engagementbeteiligung aufweisen. Weiterhin sind die Unterschiede zwischen Menschen verschiedener Bildungsgruppen seit 1999 gewachsen: Das anteilig große 
Engagement von Menschen mit hoher Bildung ist in den letzten zwanzig Jahren deutlich gestiegen, während das Engagement von Menschen mit niedriger Bildung in derselben Zeit auf niedrigerem Niveau stagniert. Die Unterschiede zwischen Menschen mit und ohne Migrationshintergrund sind zwischen 2014 und 2019 stabil geblieben. Es bleibt somit festzuhalten, dass längst noch nicht alle Bevölkerungsgruppen in gleichem Maße im Rahmen freiwilligen Engagements an der Gesellschaft teilhaben und sich in die Gesellschaft einbringen können. Sollen Ungleichheiten im Zugang zum freiwilligen Engagement und in dessen Ausgestaltung ausgeglichen werden, wäre es notwendig, entsprechende engagementpolitische Maßnahmen auszubauen.

\subsection{Demokratieförderung durch freiwilliges Engagement}

Freiwilliges Engagement, so wird in der öffentlichen und gesellschaftlichen Debatte häufig hervorgehoben, trägt zum Zusammenhalt der Gesellschaft bei und stärkt die Demokratie (Alscher et al. 2021; Ebert 2018). Dabei steht die Gemeinwohlorientierung des Engagements im Vordergrund. Wie bereits am Anfang dieses Berichtes erläutert, unterliegt die Definition des Gemeinwohls in der Regel Abstimmungs- und Aushandlungsprozessen in der Gesellschaft (siehe Kapitel 1; Deutscher Bundestag 2002). So gibt es auch freiwilliges Engagement, das zwar aus individueller Perspektive der engagierten Personen gemeinwohlorientiert ist, der Demokratie und der Gesellschaft aber letztlich auch schaden kann, beispielsweise wenn demokratiefeindliche Ziele verfolgt werden oder mit dem Engagement die Ausgrenzung einzelner Bevölkerungsgruppen angestrebt wird. Um eine Annäherung an die Zielrichtung der freiwillig Engagierten zu erreichen, wurden im Jahr 2019 Fragen zu Einstellungen zur Demokratie und zum Vertrauen in staatliche Institutionen in die Befragung des Freiwilligensurveys aufgenommen. Die Akzeptanz der Demokratie als Regierungsform und das Institutionenvertrauen sind wichtige Voraussetzungen für eine funktionierende demokratische Gesellschaft.

Die große Mehrheit der Bevölkerung in Deutschland steht der Demokratie als Regierungsform positiv gegenüber. Vergleicht man freiwillig Engagierte und Nicht-Engagierte, so zeigt sich, dass freiwilliges Engagement mit einer positiveren Einstellung zur Demokratie, einer höheren Zufriedenheit mit dem Funktionieren der Demokratie in Deutschland sowie einem stärkeren Vertrauen in gesellschaftliche Institutionen einhergeht. Die Unterschiede zwischen freiwillig Engagierten und Nicht-Engagierten sind dabei nicht durchgängig groß, aber in allen Indikatoren deutlich sichtbar. Es besteht also ein positiver Zusammenhang zwischen freiwilligem Engagement und Einstellungen gegenüber der Demokratie und demokratischen Institutionen.

$\mathrm{Ob}$ nun freiwilliges Engagement zu positiveren Einstellungen gegenüber der Demokratie führt oder ob sich Menschen mit positiveren Einstellungen zu höheren Anteilen freiwillig engagieren - diese Frage lässt sich anhand der hier vorgelegten Befunde nicht beantworten. Längsschnittstudien, die Menschen über die Zeit hinweg 
begleiten, können hierzu einen wertvollen Beitrag leisten. Es lässt sich auf Grundlage der in diesem Bericht vorgestellten Befunde aber vermuten, dass freiwilliges Engagement zu einer demokratischen und solidarischen Gesellschaft beitragen kann. Diese Interpretation stimmt hoffnungsvoll und kann dazu beitragen, Richtlinien für eine zukünftige Engagementpolitik zu formulieren. Es bedarf sowohl seitens der Politik als auch der zivilgesellschaftlichen Organisationen einer kontinuierlichen Förderung sowie Aufrechterhaltung und Weiterentwicklung von Programmen, die demokratisches Verständnis und demokratisches Handeln fordern und fördern.

\subsection{Die Erfassung freiwilligen Engagements vor dem Hintergrund des gesellschaftlichen Wandels}

Freiwilliges Engagement hat sich in den letzten zwanzig Jahren gewandelt. Es ist nicht nur der Anteil engagierter Personen gestiegen, sondern auch die Ausgestaltung des freiwilligen Engagements hat sich seit der ersten Erhebung des Freiwilligensurveys im Jahr 1999 deutlich verändert. Dieser gesellschaftliche Wandel muss bei einer langfristigen Beobachtung des freiwilligen Engagements beachtet werden. Bei der Erfassung des freiwilligen Engagements muss die Kontinuität gewahrt werden, um eine vergleichbare Basis über die Zeit hinweg zu erhalten. Gleichzeitig muss die Erfassung freiwilligen Engagements angepasst werden, um neue Themen und Formen des Engagements fassen zu können und somit dem gesellschaftlichen Wandel Rechnung zu tragen. Zudem muss der aktuelle Stand der Forschungsmethodik in die Überlegungen zu Kontinuität und Weiterentwicklung einbezogen werden. Vor dem Hintergrund dieser Überlegungen wurde das Vorgehen im Freiwilligensurvey in den letzten Jahren in drei zentralen Aspekten angepasst:

Aufnahme von neuen Themenfeldern in den Freiwilligensurvey. Für eine angemessenere Beschreibung der Entwicklung des freiwilligen Engagements wurde das Erhebungsinstrument des Freiwilligensurveys fortlaufend angepasst (siehe auch Kapitel 1; Simonson et al. 2017: 6o ff.). Auch im Jahr 2019 wurden neue Befragungsthemen in den Freiwilligensurvey aufgenommen. Es wurden erstmals Fragen zu Einstellungen zur Demokratie, zum Engagement von Geflüchteten und für Geflüchtete, zu Formen der Internetnutzung im Rahmen der freiwilligen Tätigkeit sowie zu den Spendenzwecken gestellt.

Methodische Anpassungen bei der Erfassung des freiwilligen Engagements. Im Freiwilligensurvey 2019 (wie auch schon im Freiwilligensurvey 2014) wurden die Fragen zu Aktivitäten und Engagement unter Verwendung eines klar definierten Zeitfensters (,in den letzten zwölf Monaten“) gestellt, damit Befragte über den gleichen zeitlichen Bezugsrahmen Auskunft geben und um saisonales Engagement besser zu erfassen. In den ersten drei Erhebungswellen gab es keinen konkret vorgegebenen Zeitrahmen; 
es wurde nach dem Engagement „derzeit" gefragt. Außerdem wurde im Freiwilligensurvey 2019 ein Kriterienkatalog zur Prüfung von den Angaben der Befragten zu ihren freiwilligen Tätigkeiten eingesetzt, der im Rahmen des Freiwilligensurveys 2014 erstellt und veröffentlicht wurde (siehe Kapitel 2). Unter Verwendung des Kriterienkatalogs lässt sich, im Vergleich zu den Vorwellen, transparent nachvollziehen, in welchen Fällen die Angaben zu den freiwilligen Tätigkeiten der Definition des freiwilligen Engagements widersprechen und daher von, engagiert ${ }^{\star} \mathrm{zu}$,nicht-engagiert ${ }^{\mathrm{c}}$ recodiert werden.

Ergänzung des Gewichtungsfaktors Bildung bei der Gewichtung von Daten. Personen aus unterschiedlichen Bildungsgruppen nehmen mit unterschiedlicher Wahrscheinlichkeit an Umfragen teil; daher sind verschiedene Bildungsgruppen in den Daten über- beziehungsweise unterrepräsentiert. Insbesondere Personen mit höherer Bildung sind eher bereit an Befragungen teilzunehmen als Personen mit niedrigerer Bildung (Engel \& Schmidt 2019). Da Menschen mit höherer Bildung anteilig häufiger freiwillig engagiert sind als Menschen mit niedriger Bildung, wurden durch die Nicht-Berücksichtigung der Bildung in der Datengewichtung in den bisherigen Berichten zum Freiwilligensurvey die Engagementquoten zwischen 1999 und 2014 überschätzt. Obwohl das Problem der bildungsbezogenen Stichprobenverzerrung seit der ersten Befragung des Freiwilligensurveys im Jahr 1999 besteht, wurde es zum ersten Mal im Zuge des Berichts zur Erhebung 2014 diskutiert. Mit der Berichtslegung zum Freiwilligensurvey 2019 werden die Daten aller Erhebungswellen des Freiwilligensurveys erstmals für alle Analysen auch nach Schulbildung gewichtet, damit ein angemesseneres Bild des freiwilligen Engagements in der Bevölkerung gewährleistet werden kann (siehe Kapitel 2).

Im Freiwilligensurvey wurden somit Anpassungen im Sinne der eingangs genannten Überlegungen - kontinuierliche Erfassung des Engagements, Einbezug neuer Themenfelder sowie Berücksichtigung des Stands der Forschungsmethodik - vorgenommen. Dabei gilt: Methodische Anpassungen, die angesichts der genannten Überlegungen inhaltlich sinnvoll und notwendig sind, können sich auf die Schätzung der Engagementquote und andere Ergebnisse auswirken (siehe auch Kapitel 3).

\subsection{Implikationen und Ausblick}

Für eine Engagementpolitik, die gleichwertige Teilhabechancen für alle ermöglichen soll, aber auch für die zivilgesellschaftlichen Organisationen, die die Vielfalt der Gesellschaft abbilden wollen, gibt es noch einiges zu tun. Die Ermöglichung gleichwertiger Teilhabechancen könnte für die Zivilgesellschaft eine Gelegenheit bieten, mehr freiwillig Engagierte für ein dauerhaftes Engagement gewinnen und binden zu können. Dass dies eine gesamtgesellschaftliche Aufgabe ist, wird bei der Frage der Ge- 
schlechtergerechtigkeit deutlich: Eine gleichwertige Ausgestaltung im Engagement kann nur dann gelingen, wenn es auch in anderen gesellschaftlichen Bereichen eine gerechte Arbeitsaufteilung gibt. Darüber hinaus zeigt sich mit Blick auf die ungleichen Zugangschancen verschiedener Bildungsgruppen: Um die Teilhabechancen im Engagement gleichermaßen zu gestalten, müssen beispielsweise der Zugang zu Informationen und die Möglichkeiten der Nutzung digitaler Technologien verbessert werden. Aufgabe der Engagementpolitik ist es, Rahmenbedingungen für freiwilliges Engagement zu schaffen und allen Mitgliedern der Gesellschaft die Möglichkeit für gesellschaftliche Teilhabe zu bieten, um auf diese Weise eine solidarische, offene und demokratische Gesellschaft zu stützen.

Wie wird sich freiwilliges Engagement in den nächsten Jahren weiter entwickeln? Diese Frage lässt sich allein auf der Grundlage eines Surveys, der eine Momentaufnahme darstellt, nicht beantworten. Mit dem Beginn der Corona-Pandemie im Jahr 2020 kam es zu weitreichenden wirtschaftlichen und gesellschaftlichen Einschnitten, die auch 2021 weiterhin bestehen und auch darüber hinaus Nachwirkungen haben werden. Hiervon ist auch die Zivilgesellschaft grundlegend betroffen. Welche langfristigen Folgen die Corona-Pandemie für das freiwillige Engagement haben wird, lässt sich derzeit noch nicht abschließend beantworten. Zu erwarten sind unter anderem Veränderungen hinsichtlich der Bedeutung des Internets für das freiwillige Engagement. Auch könnte sich der Fokus bei Zielstellungen und Zielgruppen ändern, möglicherweise bis hin zur Entstehung neuer Formen freiwilligen Engagements. Darüber hinaus wird sich zeigen, wie sich die Beteiligungsquoten und Zugänge freiwilligen Engagements weiter entwickeln werden. Der Freiwilligensurvey 2019, der vor Beginn der Corona-Pandemie erhoben wurde, kann im Zusammenhang mit ergänzenden Erhebungen einen Vergleichsanker für Aussagen hierzu bilden.

\section{Literatur}

Alscher, M., Priller, E., \& Burkhardt, L. (2021). Zivilgesellschaftliches Engagement. In: Statistisches Bundesamt (Destatis), Wissenschaftszentrum Berlin für Sozialforschung (WZB) \& Bundesinstitut für Bevölkerungsforschung (BiB) (Hrsg.) Datenreport 2021. Ein Sozialbericht für die Bundesrepublik Deutschland (S. 399-407). Bonn: Bundeszentrale für politische Bildung.

Beigang, S., Fetz, K., Foroutan, N., Kalkum, D., \& Otto, M. (2016). Diskriminierungserfahrungen in Deutschland. Erste Ergebnisse einer repräsentativen Erhebung und einer Betroffenenbefragung. Berlin: Antidiskriminierungsstelle des Bundes. Online: https://www.antidiskriminierungsstelle.de/SharedDocs/Downloads/DE/publikationen/Expertisen/expertise_diskriminierungserfahrungen_in_deutschland.pdf?_blob=publicationFile\&v=7 (zuletzt abgerufen: 26. 02. 2021).

Deutscher Bundestag (2002). Bericht der Enquete-Kommission „Zukunft des Bürgerschaftlichen Engagements“. Bürgerschaftliches Engagement: auf dem Weg in eine zukunftsfähige Bürgergesellschaft (Drucksache 14/8900). Berlin: Deutscher Bundestag. 
Düx, W., Prein, G., Sass, E., \& Tully, C. (2009). Kompetenzerwerb im freiwilligen Engagement. Eine empirische Studie zum informellen Lernen im Jugendalter. Wiesbaden: VS Verlag.

Ebert, O. (2018). Engagiert für Demokratie - Herausforderung und Perspektiven in Ostdeutschland. BBE-Newsletter Nr. 24. Online: https://www.b-b-e.de//fileadmin/Redaktion/o5_ Newsletter/01_BBE_Newsletter/2018/newsletter-24-ebert.pdf (zuletzt abgerufen 17.03. 2020).

Engel, U. \& Schmidt, B.-O. (2019). Unit- und Item-Nonresponse. In: N. Baur \& J. Blasius (Hrsg.) Handbuch Methoden der empirischen Sozialforschung (S. 385-404). Wiesbaden: Springer VS.

Roth, R. (2020). Demokratie und Bürgerbeteiligung in Zeiten von COVID-19. (Opuscula, 141). Berlin: Maecenata Institut für Philanthropie und Zivilgesellschaft.

Schirilla, N., \& Schramkowski, B. (2012). Junge Menschen mit Migrationshintergrund im Freiwilligen Sozialen Jahr: Erfolgsfaktoren und Barrieren. In M. Schmidle, B. Schramkowski \& U. Slüter (Eds.), Integration durch Mitmachen: FSJ für junge Menschen mit Migrationshintergrund. Freiburg: Lambertus-Verlag.

Simonson, J., Hameister, N., \& Vogel, C. (2017). Daten und Methoden des Deutschen Freiwilligensurveys. In: J. Simonson, C. Vogel \& C. Tesch-Römer (Hrsg.) Freiwilliges Engagement in Deutschland - Der Deutsche Freiwilligensurvey 2014 (S. 51-88). Wiesbaden: Springer VS.

Vogel, C., Simonson, J., \& Tesch-Römer, C. (2017). Freiwilliges Engagement und informelle Unterstützungsleistungen von Personen mit Migrationshintergrund. In: J. Simonson, C. Vogel \& C. Tesch-Römer (Hrsg.) Freiwilliges Engagement in Deutschland - Der Deutsche Freiwilligensurvey 2014 (S. 601-634). Wiesbaden: Springer VS.

Open Access Dieses Kapitel wird unter der Creative Commons Namensnennung Nicht kommerziell 4.0 International Lizenz (http://creativecommons.org/licenses/bync/4.0/deed.de) veröffentlicht, welche die nicht-kommerzielle Nutzung, Vervielfältigung, Bearbeitung, Verbreitung und Wiedergabe in jeglichem Medium und Format erlaubt, sofern Sie den/die ursprünglichen Autor(en) und die Quelle ordnungsgemäß nennen, einen Link zur Creative Commons Lizenz beifügen und angeben, ob Änderungen vorgenommen wurden.

Die in diesem Kapitel enthaltenen Bilder und sonstiges Drittmaterial unterliegen ebenfalls der genannten Creative Commons Lizenz, sofern sich aus der Abbildungslegende nichts anderes ergibt. Sofern das betreffende Material nicht unter der genannten Creative Commons Lizenz steht und die betreffende Handlung nicht nach gesetzlichen Vorschriften erlaubt ist, ist auch für die oben aufgeführten nicht-kommerziellen Weiterverwendungen des Materials die Einwilligung des jeweiligen Rechteinhabers einzuholen. 